\section{MS4-O5 How well do we understand macromolecular crystals?}

Andrea Thorn ${ }^{1}$, Rob Nicholls ${ }^{1}$, Garib Murshudov ${ }^{1}$

1. MRC Laboratory of Molecular Biology, Francis Crick Avenue, Cambridge Biomedical Campus, Cambridge CB2 0QH, UK.

email: andrea.thorn@web.de

In X-ray structure determination, the R-value reports how well a model agrees with the experimental data. In small molecule crystallography, R-values of $3 \%$ are routinely reached. However, for biological macromolecules, R-values are normally around $20 \%$ and even when the data extend to atomic resolution, the average $\mathrm{R}$-value is $14 \%$.

This is clear evidence that something is amiss; our current models of macromolecular crystal structures seem to be lacking. But what is this difference between our models and reality and how would we observe this difference, given that our assumptions about macromolecular structures are used to generate phases (and hence electron density) in the first place?

In this talk, some answers to these questions will be presented. It will also be shown how model deficiencies hamper in particular the structure determination of problematic structures, such as membrane proteins and large macromolecular complexes. In these cases, only low resolution data might be available and the poor phase estimates currently obtainable result in noisy electron density maps. A better understanding of the shortcomings in our current models (and methods) could be an important factor to solving the most difficult structures and to improving the others.

Keywords: macromolecules, refinement, phasing, macromolecular crystals
MS5. Structure and function of

enzymes

Chairs: Joel Sussman, Ute Krengel

MS5-O2 New structures of Thermotoga

maritima integral membrane

pyrophosphatase suggest a conserved coupling mechanism for proton and sodium transport

Adrian Goldman ${ }^{1,2,3}$, Juho Kellosalo ${ }^{2}$, Craig Wilkinson ${ }^{1}$, Tommi Kajander $^{2}$, Yu-Juh Sun ${ }^{4}$

1. Astbury Centre of Structural Molecular Biology, University of Leeds, Leeds, United Kingdom

2. University of Helsinki, Helsinki, Finland

3. Nankai University, Tianjin, China

4. National Tsing Hua University, Hsinchu, Taiwan

email: a.goldman@leeds.ac.uk

Membrane-bound pyrophosphatases couple transport of protons and/or sodium-ions through the biological membrane to pyrophosphate hydrolysis or synthesis (1). They occur in plants and in unicellular organisms and are crucial for abiotic stress tolerance (2). mPPases are dimeric, with both monomers having 16 trans-membrane helices (TMHs) and one continuous active site with distinctive regions for PP binding and ion transport (3). $\mathrm{PP}_{\mathrm{i}}$ binding drives the closure of the cytoplasmic active site and thus mPPases utilize an alternate-access mechanism for ion pumping. PPi hydrolysis is carried by an activated water molecule, which is coordinated between two conserved aspartates (4). Two new structures of different membrane-bound pyrophosphatases: a sodium-pump from Thermotoga maritima with bound sodium and competitive inhibitor, imidodiphosphate (IDP), and the Vigna radiata proton-pump with one phosphate bound reveal the movements of inner ring and outer ring TMHs and the corresponding opening and closing of the binding pocket. The structures reveal a hitherto unknown sodium-binding site and confirm our earlier hypothesis that the electrophilic phosphate group is released first from the active site $(3,5)$. Together with recent functional data, the structures also confirm our earlier hypothesis that TMH12 movement plays a role in ion pumping, likely by driving the formation of the periplasmic/lumenal exposed conformation of the enzyme. The structures also suggest a model for how the coupling between ion pumping and PP. hydrolysis is ensured by movement of TMHs 6 and 16, which brings the conserved aspartates into the correct position for catalytic water coordination only in the ion-bound state of the enzyme. These changes allow us to propose how proton-pumping evolved from sodium-pumping in mPPases.

References 